$\mathrm{FN}-394$

1100.600

Submitted to Health Physics

NEUTRON SKYSHINE MEASUREMENTS AT FERMILAB

J. D. Cossairt and L. V. Coulson

November 1983 


\title{
NEUTRON SKYSHINE MEASUREMENTS AT FERMILAB
}

J. D. Cossairt and L. V. Coulson

Fermi National Accelerator Laboratory*

Batavia, Illinois, USA 60510

\begin{abstract}
Neutron skyshine has been a significant source of environmental radiation exposure at many high energy proton accelerators. A particularly troublesome source of skyshine neutrons has existed at Fermilab during operation of the $400 \mathrm{GeV}$ high energy physics program. This paper reports on several measurements of this source made with a De Pangher precision long counter at large distances. It is shown that the spatial distribution of the neutron skyshine can be adequately described for radiation protection purposes by phenomenological formulas developed by other workers. With due care in calibration procedures, the absolute magnitude of the distributions may be matched directly to the more conventionally measured absorbed dose distribution near the source.
\end{abstract}

*Operated by Universities Research Association: Inc. under contract with the U. S. Department of Energy. 


\section{INTRODUCTION}

Neutron skyshine from thinly shielded high energy proton accelerators has been known to be a problem since the early $1950^{\prime} \mathrm{s}$ especially in situations in which accelerators had little or no roof shielding (Pa73). Various attempts have been made to describe in theoretical or phenomenological ways both the correlation of the intensity of the skyshine radiation with the neutron emission or absorbed dose rate at the source and the dependence of this intensity with distance from the source. Patterson and Thomas (Pa73) give an overview of the skyshine problem while Rindi and Thomas (Ri75) and Stevenson and Thomas (St83) give more detailed accounts of present results for accelerators with proton energies up to $30 \mathrm{GeV}$. References (Pa73) and (Ri75) give the following empirical expression which adequately describes the existing data for radial distances greater than 50m:

$$
\phi(r)=\frac{a Q}{4 \pi r^{2}}\left(I-e^{-r / \mu}\right) e^{-r / \lambda}
$$

In this expression $a=2.8$ and represents an empirical "buildup" factor, $r$ is the radial distance from the source to the detector in meters, and $\mu$ is a "buildup" relaxation 
length usually taken to be 56 meters. $Q$ is the source strength which must be dimensionally consistent with $\phi(r)$. Thus, if $\phi(r)$ is a measurement of neutrons $\mathrm{m}^{-2} \mathrm{sec}^{-1}$, then $Q$ would have to be neutrons $\sec ^{-1}$. A measurement of mrads $\mathrm{hr}^{-1}$ at radius $\mathrm{r}$ would indicate $Q$ to be in units of $\mathrm{mrad}-\mathrm{m}^{2} \mathrm{hr}^{-1}$. Eq. (1) is most correct for isotropic or nearly isotropic sources viewed at distances large compared with the source dimensions. In this formula $\lambda$ is an attenuation length which is a parameter of the energy spectrum involved.

This equation is justified by application to the existing data with values of $\lambda$ typically varying between 250 and 990 meters. A value of 830 meters $1100 \mathrm{~g} / \mathrm{cm}^{2}$ of air at NTP) would correspond to the interaction length of $100 \mathrm{MeV}$ neutrons which Iikely control the propagation of the hadronic cascade in air. Values of $\lambda$ larger than 830 meters are possible if the spectrum contains neutrons of very high energy (E > $150 \mathrm{MeV}$ ) (Pa73). For example, values as large as $1300 \mathrm{~m}$ were reported by Miyayima, et al at the KEK synchrotron (Mi77).

Stevenson and Thomas (St83) have developed a concise formula which may be used to directly obtain dose equivalents at radial distances $r$ from an isotropic source:

$$
H(r)=\frac{3 \times 10^{-13}}{r^{2}} \Omega e^{-r / \lambda}
$$


where $H(r)$ is the dose equivalent (rem) at radial distance $r$ (meters) for $\&$ neutrons emitted by the source. The parameter $\lambda$ has the same meaning as in Eq (1).

\section{THE SOURCE}

The skyshine source studied in the present work has a very complicated geometry which is shown in Figure 1. Figure 2 shows a map of the larger area of the Fermilab site surrounding the source and indicates the routes along which surveys were made. At the source an incident beam of $400 \mathrm{GeV}$ protons strikes a $0.3 \mathrm{~m}$ long beryliium target (approximately one interaction length for high energy protons) in a large steel target box. Approximately 5 meters downstream the charged particles are magnetically deflected into a massive primary beam dump. The neutral particles,primarily neutrons and photons, produced by proton interactions in the target, continue downstream about 70 meters where they are absorbed by a neutral beam dump. The neutral beam intensity is about one percent of incident proton intensity. About 12 meters downstream of the beryllium target the neutral beam passes through a lead converter (3 to $6 \mathrm{~mm}$ thick) which converts some of the photons in the beam into electrons. The beam of electrons (about $10^{-6}$ of the incident proton beam intensity) is magnetically steered through a channel in the neutral dump and delivered to a physics experiment 
located 250 meters downstream of the primary target. It should be noted that the proton beam is located about 6 meters below ground level.

Concrete shielding was provided inside the enclosure and some was stacked on the roof. The effectiveness of the shielding was reduced because of numerous architectural compromises. As is shown on Figure 1 , shielding was added to the problem area in 1979. Nominal primary proton beam intensity is about $3 \times 10^{12}$ protons in a one second long beam spill every 12 seconds or $10^{15}$ protons per hour. All quantities of interest (counts $\mathrm{hr}^{-1}$, mrads $\mathrm{hr}^{-1}$, etc.) are normalized to this intensity. Measurement of the absorbed dose rate near this source is complicated by the presence of muons as well as neutrons. This muon "contamination" is more pronounced at the more forward angles with respect to the incident proton beam. These two components were separated by measuring the total absorbed dose rate with a tissue equivalent ion chamber and subtracting the absorbed dose rate contributed by muons as measured with a scintillation counter telescope $(\operatorname{Cos} 3)$. Figures 3 and 4 indicate the neutron absorbed dose measurements above the source before and after the concrete shielding shown in Figure $I$ was added. In 1980 the fraction of the total absorbed dose due to neutrons was measured to be 0.3 and 1.0 at locations $C A$ and $C B$, respectively, indicated on Figure 4. The value 
of this fraction at intermediate points was obtained by linear interpolation. Because of improvements in survey techniques and instrumentation the 1980 survey data should be regarded as the more reliable. The errors in measuring neutron absorbed dose at high energies are the standard ones perhaps best described by Höfert and Raffnsфe (Ho80). It should be noted that the source is rather extensive and is asymmetric; being very close to the western wall.

\section{MEASUREMENT TECHNIQUE}

The principle measurements of this work were made using a DePangher precision long counter (De66) mounted in a vehicle equipped with standard commercial counting electronics which were gated in synchronization with the accelerator cycle through the use of a microwave telemetry system to alternately obtain beam-on and, between accelerator cycles, background data. The telemetry also transmitted beam intensity information.

A plutonium-beryllium source calibrated by the U. S. National Bureau of Standards was used to set up the long counter electronics. A ${ }^{10} \mathrm{BF}_{3}$ proportional counter was used in the long counter. Thus a simple discriminator arrangement sufficed to distinguish thermal neutron capture events due to the ${ }^{10_{B}}(n, \alpha){ }^{7} \mathrm{~L}$ reaction from gamma ray or muon induced events. 
The ratio of long counter counts to neutron absorbed dose was measured at locations $\mathrm{CA}$ and $\mathrm{CB}$ shown in Figure 4. The results were:

$$
\begin{aligned}
& \mathrm{CA}: 30600 \text { long counter counts } / \mathrm{mrad} \\
& \mathrm{CB}: 53600 \text { long counter counts/mrad }
\end{aligned}
$$

The smaller calibration factor at $C A$ is to be expected because of the higher energy neutron spectrum encountered at the more forward angles relative to the incident proton beam.

\section{DATA AND ANALYSIS}

Figures 5-7 show the data collected where error bars represent one standard deviation counting statistics. The data collected in 1975 along routes 1 and 3 in Figure 2 is useful in checking the dependence of the skyshine distribution upon "scattering angle" relative to the incident beam direction. A number of data points were collected at radial distances within 15 percent of 120 meters covering a large range of polar angles $\theta$ from the incident beam direction and plotted as an angular distribution in Figure 5. A large forward peak having a full width at half maximum of $3.8^{\circ}$ is superimposed on a roughly isotropic distribution at this radius. This peak is most likely explained as direct neutrons emerging from the enclosure rather than skyshine neutrons because the FWHM of the distribution extrapolates to a width 
comparable to that of the source enclosure at its downstream end. This forward peak is not seen at larger radial distances at the measurement height 1.2 meters above ground level. Away from the forward peak the distribution levels off with a higher intensity on the east side than on the west side as expected because of the asymmetry of the source (i.e., locations on the west side are better shielded or shadowed by the west wall). The skyshine angular distribution is best described as an isotropic one with a forward peak superimposed on it.

Figures 6 and 7 show the measured skyshine distributions plotted as $r^{2} \phi(r)$ to remove the $1 / r^{2}$ dependence and exhibit the residual variations. The ordinate is thus in units of counts $-m^{2} \mathrm{hr}^{-1}$. Count rates at large radii were very low resulting in larger errors. The source asymmetry is again seen in Figure 6 .

Equation (1) was fitted to the data assuming the buildup parameters a and $\mu$ to be correct and using linear least squares fits to obtain values of $Q$ and $\lambda$. These fitted parameters are indicated above the apptopriate distribution and the solid line shows the fitted function. The very large values of $\lambda$ (up to $1200 \mathrm{~m}$ ) obtained here for the measurements out to large radii (r $>500 \mathrm{~m}$ ) indicate that high energy $(E>150 \mathrm{MeV})$ neutrons are propogating the hadronic cascade in the air. Measurements above the source using a multisphere technique also 
indicate the neutron energy spectrum to have a substantial, though imprecisely defined, high energy component $(\mathrm{Gr} 81)$. If one only includes data taken at $r<$ 500 meters in the least squares analysis, smaller values of $\lambda$ (about $350 \mathrm{~m}$ ) result. This may indicate that the neutron energy spectrum seen at ground level is approaching equilibrium with increasing $r$ with the corresponding change in the value of $\lambda$.

It is interesting to compare the value of the source strength $Q$ obtained from fitting Eq (1) to the skyshine measurements with the value one obtains by integrating the neutron absorbed dose rates shown in Figures 3 and 4 over the area of the source. To make this comparison one must convert the areal integrals from mrad-m $\mathrm{m}^{-1}$ to counts $-\mathrm{m}^{2} \mathrm{hr}^{-1}$. For the 1980 data, the conversion factors (CA and CB) were used (with linear interpolations for intermediate locations). For the less reliable 1975 measurements, 50000 counts $\mathrm{mrad}^{-1}$ was used. Table I shows the neutron absorbed dose rate areal integrals (in mrad $-\mathrm{m}^{2} \mathrm{hr}^{-1}$ and in counts $-\mathrm{m}^{2} \mathrm{hr}^{-1}$ ) and the value of $Q$ obtained by fitting the skyshine data to Eq(1). The agreement is quite good, given the complexity of the geometry.

It is instructive here to compare Eqs (I) and (2). In view of the limited knowledge of the details of the neutron spectrum, an average quality factor of five was assigned to it following Patterson, et.al(Pa71). Applying 
this to Table $l$ of Stevenson and Thomas (St83), one can obtain values of neutron flux per unit of absorbed dose for $1 / E$ neutron spectra having various maximum energies $E_{\max }$. For $E_{\max }=1.0 \mathrm{Gev}$, which may be reasonable in this case, this procedure results in a value of $3.1 \times 10^{9} \mathrm{n}-\mathrm{m}^{-2}$ mrad ${ }^{-1}$. Choosing $E_{\max }$ to be 0.1 or $10.0 \mathrm{GeV}$ would result in absorbed dose to flux conversion factors of $4.6 \times 10^{9}$ or $1.4 \times 10^{9} \mathrm{n}-\mathrm{m}^{-2} \mathrm{mrad}^{-1}$, respectively, so that the exact choice of $\mathrm{E}_{\max }$ over the range from 0.1 to $10 \mathrm{GeV}$ results in at most a factor of 3 discrepancy. Using the value for $\mathrm{E}_{\max }=1.0 \mathrm{GeV}$ and the assumption of a quality factor of five we can rewrite $\mathrm{Eq}(1)$ as follows:

$$
D(r)=\frac{0.19}{r^{2}} Q e^{-r / \lambda}
$$

where $D(r)$ is absorbed dose rate at radial coordinate $r$ (mrad $\mathrm{hr}^{-1}$ ) and $Q$ is source strength in mrad-m $\mathrm{hr}^{-1}$. This equation is quite consistent with $\mathrm{Eq}(1)$ and hence with our measurements for large $r$ where the factor $\left(1-e^{-r / \mu}\right)$ can be ignored observing that $a / 2 \pi$ has $a$ value of 0.22 and recognizing the somewhat arbitrary assumptions made about the neutron energy spectrum. 


\section{DOSE EQUIVALENT TO PERSONNEL}

It is straightforward to estimate the dose equivalent rate to personnel in this neutron field during operations of this proton beam. Using the calibration at point $C B$ and an average quality factor of five, one obtains about $10^{4}$ long counter counts/mrem. At $r=200$ meters, one would have less than $10^{4}$ counts $\mathrm{hr}^{-1}$ or about 1 mrem $\mathrm{hr}^{-1}$ from the 1975 measurement. The additional shielding reduced this to $0.1 \mathrm{mrem} \mathrm{he^{-1 }}$ in 1980 . These areas are occupied on a long term basis only by Fermilab personnel. In the earlier measurements, even at 50 meters from the source, the dose equivalent rate was only $12 \mathrm{mrem} \mathrm{hr}^{-1}$.

The nearest permanently occupied location is at about $r=2000 \mathrm{~m}$. During the conditions of the 1975 measurements, the dose equivalent rate there was no more than 0.005 mrem/hr during such operating conditions. At these large values of $r$ the dose equivalent rate would be underestimated by a factor of about 25 if one had only collected data at values of $r<500$ meters, obtained a value of $\lambda$ from a least squares fit, and extrapoláted the dose equivalent rate to 2000 meters. Thus measurements to large radii are quite important. 


\section{CONCLUSION}

These measurements verify published parameterizations derived from experience at other accelerators. It is clear that the extra shielding installed in 1979 helped the skyshine problem by reducing the area of the source (and thus the total number of emitted neutrons). Perhaps this experience will be useful in helping the designers of other facilities evaluate and avoid such problems. The importance of roof shielding discovered in the $1950^{\circ} \mathrm{s}$ is reverified. Alas, the measurements cannot be repeated because in 1983 this obviously inadequate shield was reconstructed in order to remove a very large source of direct neutron radiation.

The authors would like to thank J. Larson for his help in maintaining the apparatus mounted in the somewhat difficult environment of a vehicle, M. Gerardi for his help in unraveling the absorbed dose survey records and D. Grobe for her calibration data. 
REFERENCES

Co83 Cossairt J. D., 1983, "Recent Muon Fluence Measurements at Fermilab", Health Physics, 45, 651.

De66 Depangher J. and Nichols L. L., 1966, "A Precision Long Counter for Measuring Fast Neutron Flux Density", Pacific Northwest Laboratory Report No. BNWL -260 .

Gr81 Griffith R.V., 1981, private communication.

Ho80 Höfert $M$. and Raffnsфe C., 1980, "Measurement of Absolute Absorbed Dose and Dose-Equivalent Response for Instruments Used Around High Energy Proton Accelerators", Nucl. Instr. and Meth, 176, 443 .

Mi77 Miyajima M. , Hirayama H. , Hozimi K. , Muira S. and Katok K. , 1977, "Measurement of Stray Neutron Doses Around KEK PS-Facility (1)", NationaI Laboratory for High Energy Physics (KEK), Oho-machi, Tsukuba-gun, Ibaraki-ken, Japan, KEK-77-17.

Pa71 patterson $\mathrm{H}$. Wade, Routti Jorma T. and Thomas Ralph H., 1971, "What Quality Factor", Health physics, 20, 517 .

Pa73 Patterson H. Wade and Thomas Ralph H., 1973, Accelerator Health Physics (Academic Press, New York), pp. $437-443$. 
Ri75 Rindi A. and Thomas R. H. , 1975, "Skyshine--A Paper Tiger?", Particle Accelerators", 7, 23.

St83 Stevenson G. R. and Thomas R. H. , 1983, "A Simple Procedure for the Estimation of Neutron Skyshine from Proton Accelerators", CERN Report HS-RP/076/PP Revised (submitted to Health Physics). 


\section{LIST OF TABLE CAPTIONS}

1. Comparison of the values of $Q$ obtained from areal integrals over the source with those obtained by fitting the skyshine data with $\mathrm{Eq}(1)$. 


\begin{tabular}{|c|c|c|c|}
\hline Measurement & $\frac{\text { Absorbed }}{\text { Areal }} \frac{\text { Intege }}{\left.\text { (mrad }-m^{2} h r-1\right)}$ & $\begin{array}{c}Q \\
\left(\text { counts }-m^{2} h r^{-1}\right)\end{array}$ & $\frac{\text { Skyshine Data }}{\left.\text { (counts }-m^{2} h r-1\right)}$ \\
\hline $1-(1975)$ & & & $1.87 \times 10^{9}$ \\
\hline $2-(1975)$ & $5.0 \times 10^{4}$ & $2.5 \times 10^{9}$ & $2.73 \times 10^{9}$ \\
\hline $3-(1975)$ & & & $2.23 \times 10^{9}$ \\
\hline $4-(1980)$ & $8.1 \times 10^{3}$ & $3.9 \times 10^{8}$ & $4.91 \times 10^{8}$ \\
\hline
\end{tabular}


LIST OF FIGURE CAPTIONS

1. Views of the skyshine source under consideration.

2. Map of the general area of the skyshine source showing dates and routes along which the surveys were made. A radius of 120 meters from the approximate center of the source is indicated.

3. Neutron absorbed dose rates $(\mathrm{mrad} / \mathrm{hr})$ shown as a function of position on top of the skyshine source in 1975. The incident beam direction is shown for reference.

4. Neutron absorbed dose rates (mrad/hr) shown as a function of position on top of the skyshine source in 1980. The incident beam direction is shown for reference. $\mathrm{CA}$ and $\mathrm{CB}$ are calibration locations referred to in the text.

5. Angular distribution of long counter measurements at a radius of 120 meters from the center of the source. The angle $\theta$ is measured relative to the direction of the incident proton beam.

6. Skyshine data from surveys 1 and 3 plotted as $\mathrm{r}^{2} \phi$ as a function of radius $r$ (meters). The solid curve is the result of the least square fit of $\mathrm{Eq}$. (1) to the route 3 data while the dashed curve is for a value of $\lambda$ of 830 meters. 
7. Skyshine data from surveys 2 and 4 plotted as $r^{2} \phi$ as a function of radius $r$ (meters). The solid curves are from the least square fit of Eq. (I) to the data points while the dashed curve is for a value of $\lambda$ of 830 meters. 

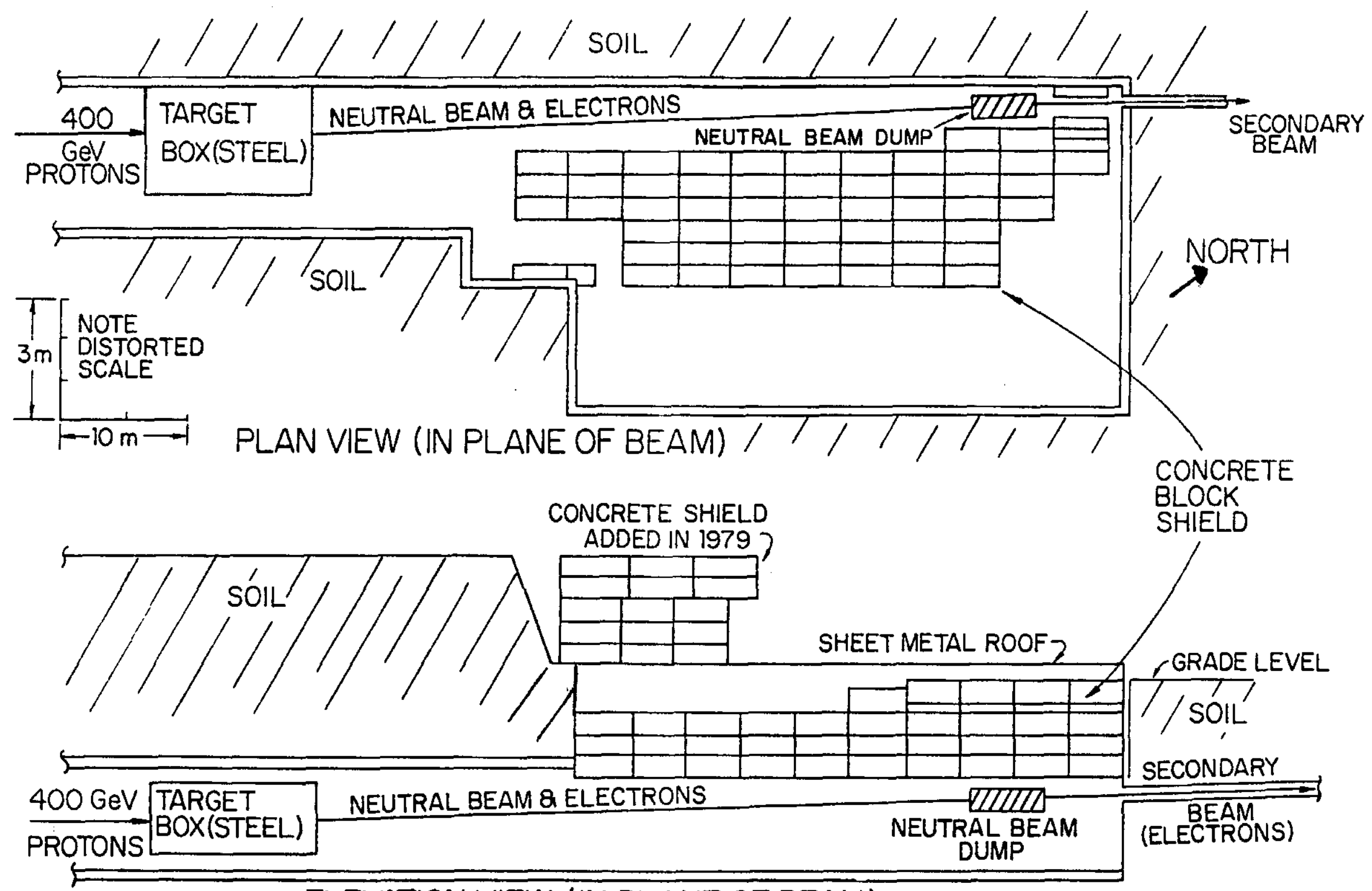

ELEVATION VIEW (IN PLANE OF BEAM)

Figure 1 


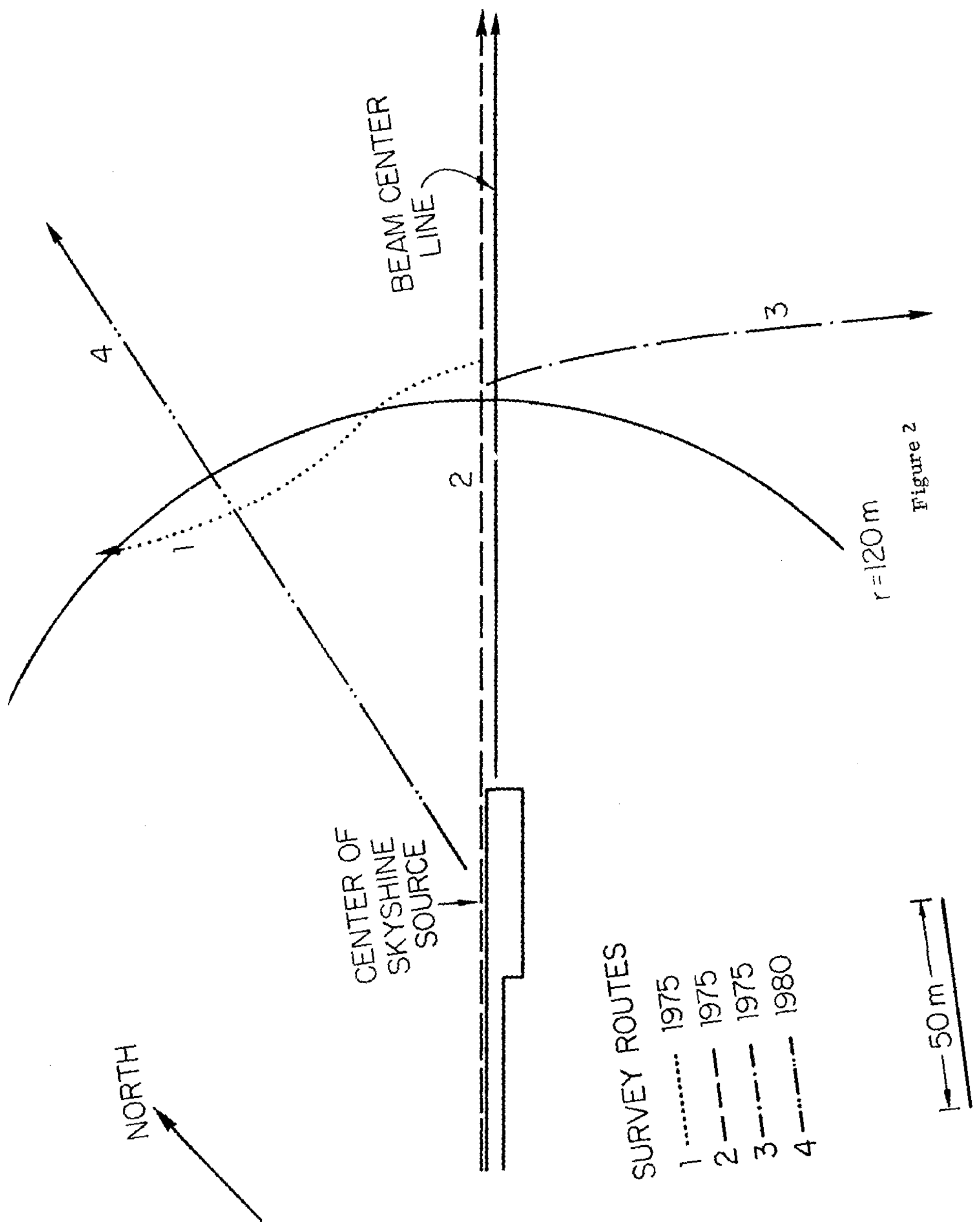




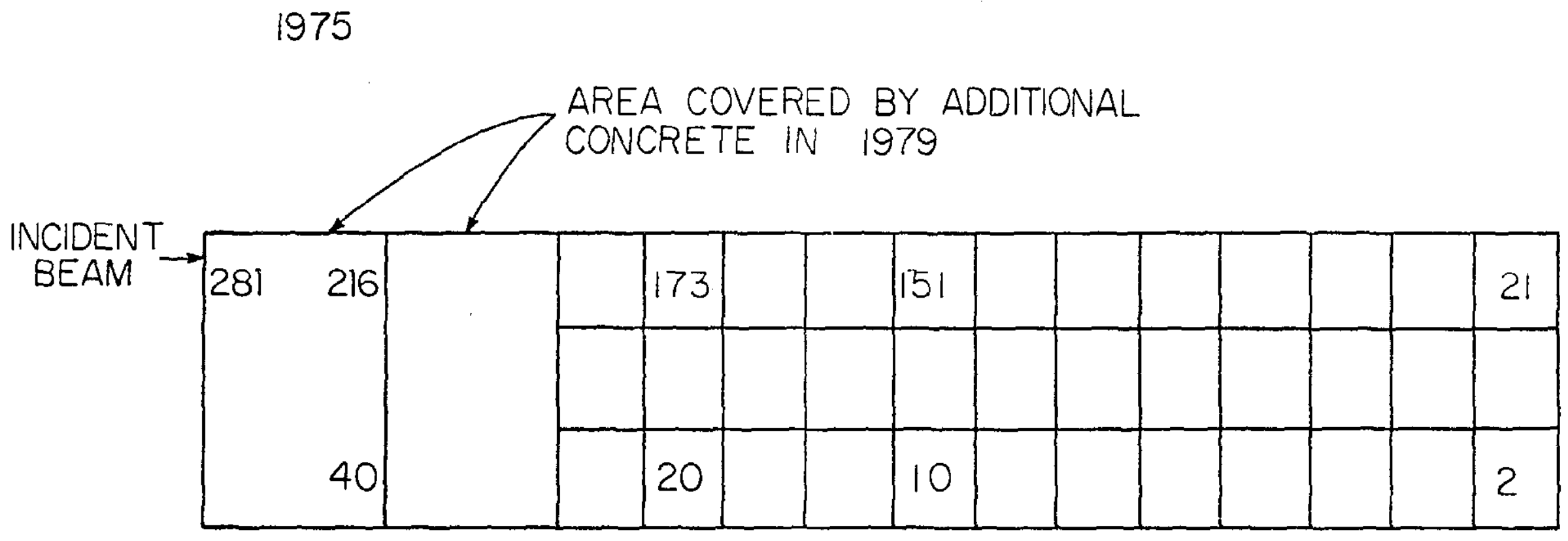

Figure 3 


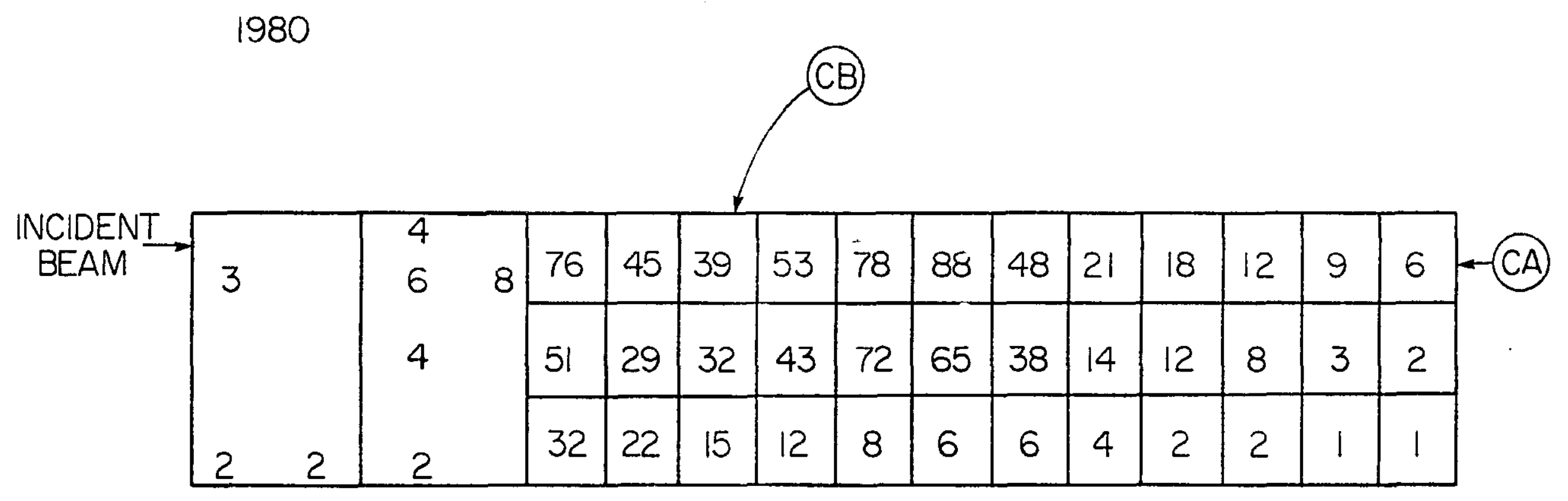

Figure 4 


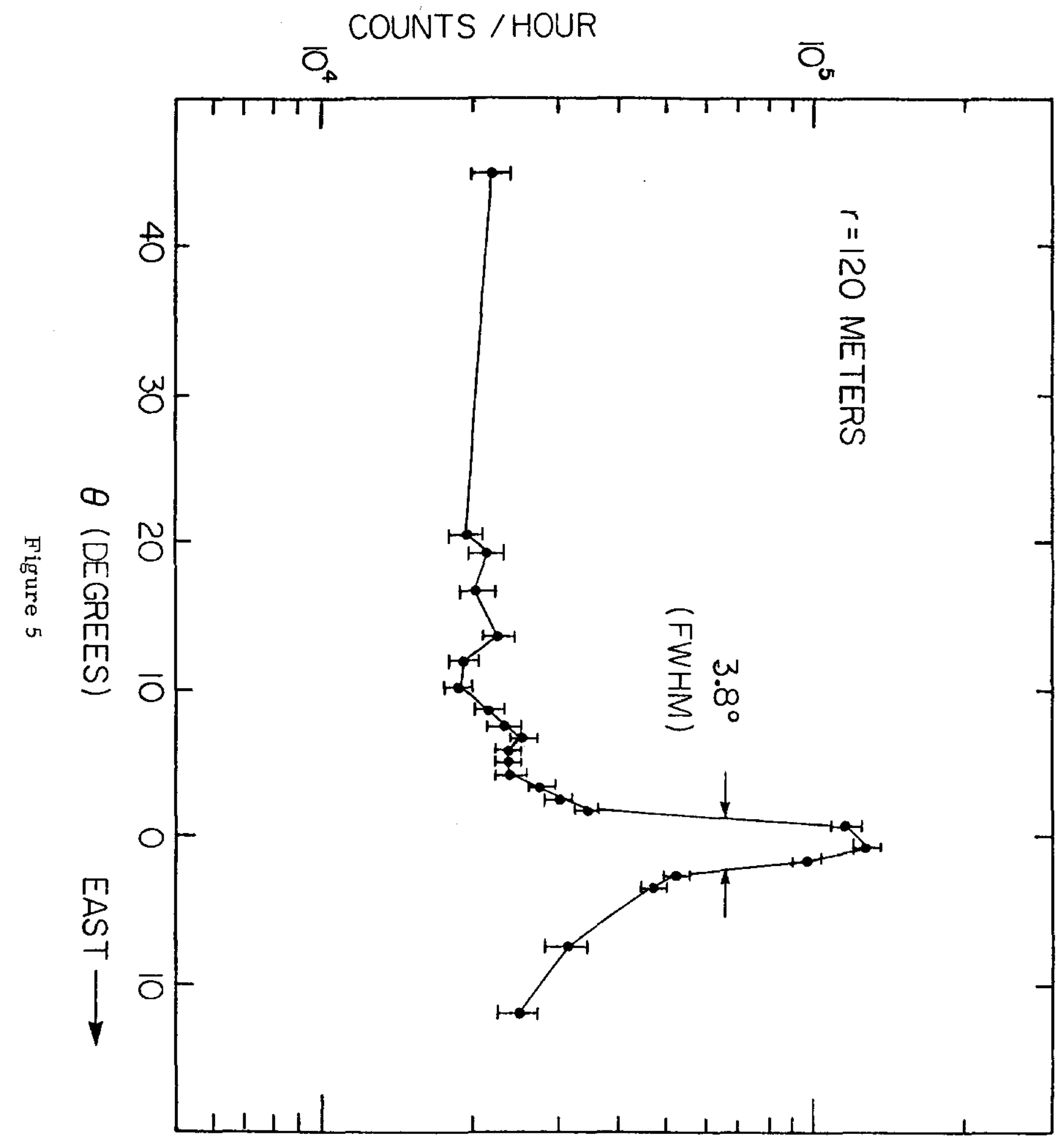




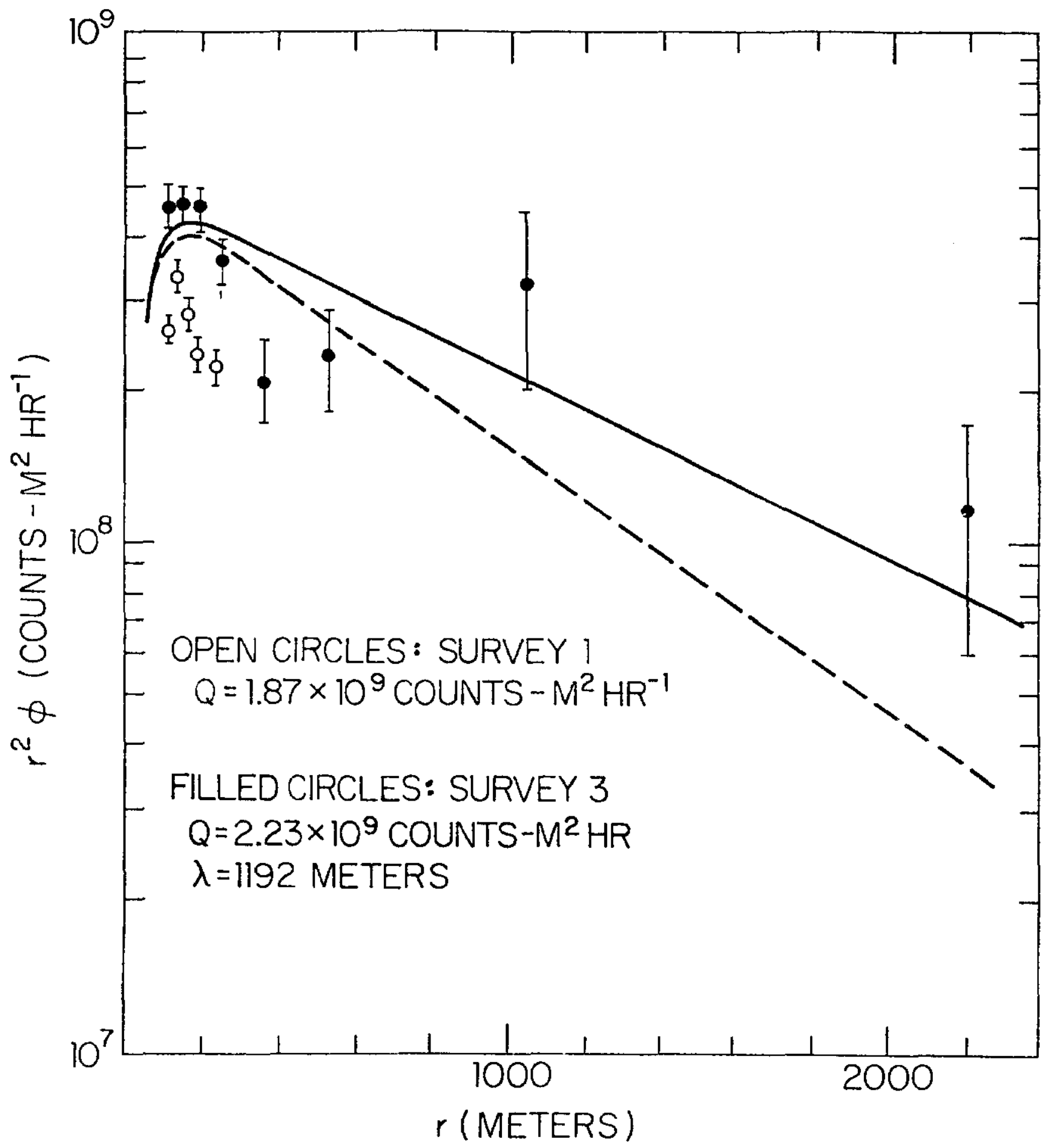

Figure 6 


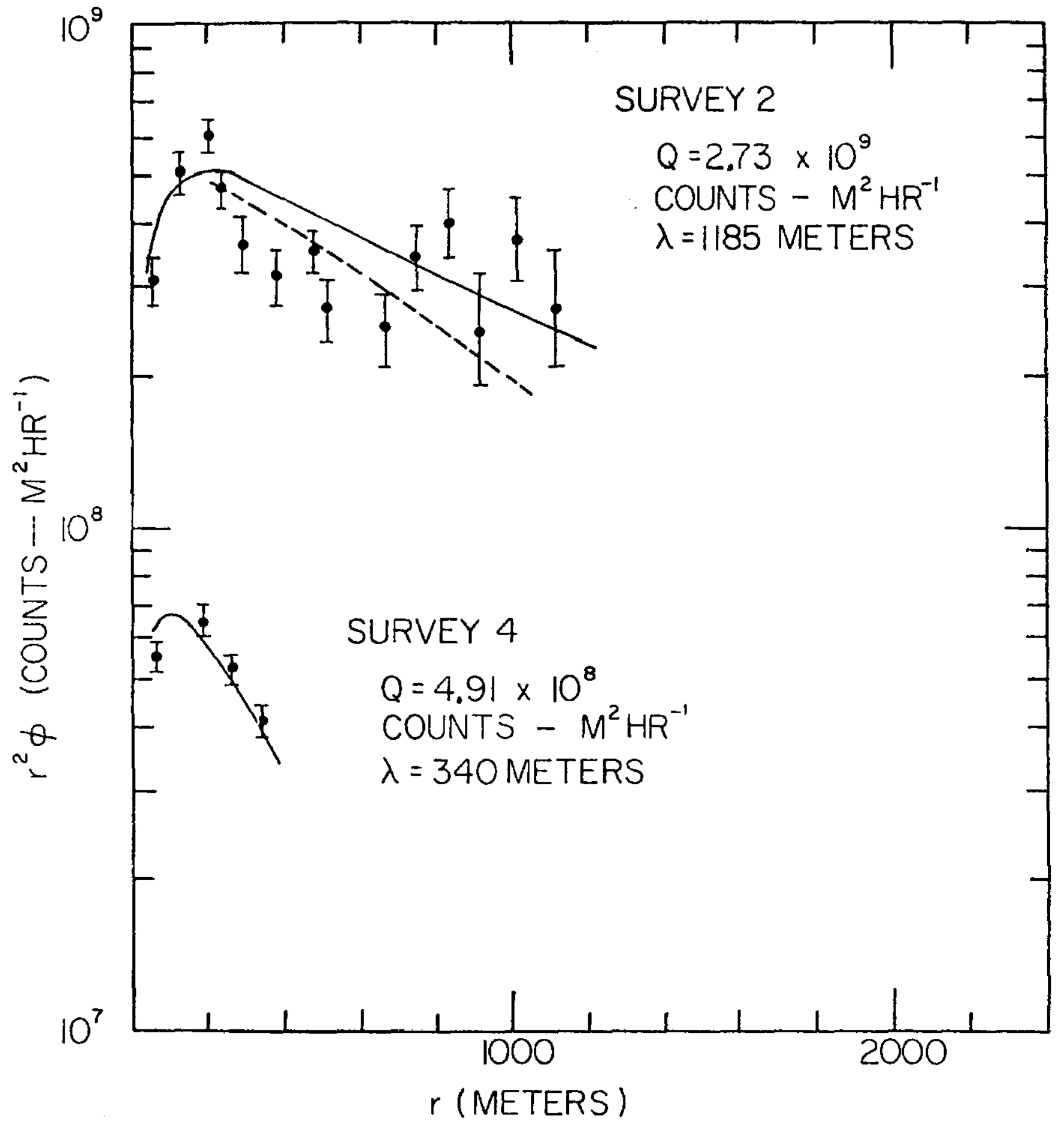

Figure 7 